\title{
Use of 68Ga-DOTATATE PET/CT imaging for staging evaluation of Neuroblastoma in the COVID 19 Pandemic: Potential for clinical
}

use

Ata Maaz ${ }^{1}$, Jim O’Doherty ${ }^{2}$, and Mehdi Djekidel ${ }^{1}$

${ }^{1}$ Sidra Medical and Research Center

${ }^{2}$ MSD Translational Medicine Research Centre

June 26, 2020

\begin{abstract}
Background: MIBG imaging has been the standard for neuroblastoma staging following its discovery 4 decades ago. Novel agents such as 18F-FDOPA and 68Ga-DOTATATE are being widely used nowadays in academic centers. In the midst of the COVID19 pandemic, procurement of MIBG has proved particularly challenging, necessitating the use of 68Ga DOTATATE positron emission tomography. Methods: 68Ga-DOTATATE PET/CT imaging was carried out for completion of staging investigation for three pediatric patients who were diagnosed with Neuroblastoma at our institution, as 123Iodine MIBG became unavailable during the pandemic. Results: 68Ga-DOTATATE PET/CT scans were carried out in all three pediatric patients without any complications. All patients showed 68Gallium DOTATATE avid disease. PET/CT scans showed better spatial resolution, increased lesion contrast and demonstrated high accuracy in staging neuroblastoma patients in concordance with current EANM guidelines. Conclusion: 68Ga-DOTATATE PET/CT imaging can be reliably used as an alternative to MIBG for staging and evaluation of patients with neuroblastoma. Impact may be best in high risk disease.
\end{abstract}

TITLE: Use of ${ }^{68}$ Ga-DOTATATE PET/CT imaging for staging evaluation of Neuroblastoma in the COVID 19 Pandemic: Potential for clinical use

SHORT RUNNING TITLE: ${ }^{68}$ Ga-DOTATATE PET/CT for Neuroblastoma Staging

Key Words: Neuroblastoma imaging, SARS-CoV-2 (COVID-19), ${ }^{68}$ Ga-DOTATATE PET/CT, ${ }^{123}$ I-MIBG

Word Count: 2118

AUTHORS: Ata Ur Rehman Maaz ${ }^{1}$; Jim O'Doherty ${ }^{2}$; Mehdi Djekidel ${ }^{3}$

Abbreviations:

\begin{tabular}{ll}
\hline${ }^{68} \mathrm{Ga}$ & Gallium \\
\hline DOTA & 1,4,7,10-tetraazacyclotetradecane- $N, N^{\prime}, N^{\prime}, N^{\prime \prime}$-tetraacetic acid \\
DOTATATE & DOTA-0-Tyr3-Octreotate \\
${ }_{\text {MIBG, }{ }^{123} \mathrm{I}-\text { MIBG, }}$ & Metaiodobenzylguanadine \\
${ }^{18} \mathrm{~F}-\mathrm{DOPA}$ & L-3,4-Dihydroxy-6-[18F]fluorophenylalanine \\
${ }^{18} \mathrm{FDG}$ & 2-deoxy-2-[fluorine-18]fluoro-D-glucose \\
$\mathrm{PET}$ & Positron Emission Tomography \\
$\mathrm{CT}$ & Computerized Tomography \\
MRI & Magnetic resonance Imaging \\
EANM & European Association of Nuclear Medicine
\end{tabular}




\begin{tabular}{ll}
\hline${ }^{68} \mathrm{Ga}$ & Gallium \\
\hline SST & Somatostatin \\
SSTR & Somatostatin Receptor \\
NET & Neuroendocrine Tumor \\
VMA & Vanillylmandellic Acid \\
HVA & Homovanillic Acid \\
SPECT & Single Photon Emission Computerized Tomography \\
GA & General Anesthesia \\
GMP & Good manufacturing practices \\
mSv & Millisievert \\
mAs & Milliampere-seconds \\
MBq & Megabecquerels \\
kVp & Kilovoltage Peak \\
MeV & Mega-electronvolt(s) \\
SCA & Segmental chromosomal abnormalities \\
NCA & Segmental chromosomal abnormalities \\
\hline
\end{tabular}

${ }^{1}$ Corresponding author:

Dr Ata Ur Rehman Maaz,

Senior Attending Physician,

Department of Pediatrics, Division of Hematology Oncology,

Sidra Medicine,

Al-Luqta Street,

PO Box No. 26999,

Doha,

Qatar.

Email: amaaz@sidra.org

\section{Co-author affiliations:}

1. Clinical Imaging Research Centre, Centre of Translational Medicine (MD6), 14 Medical drive, \#B1-01, Singapore117599.

2. Department of Diagnostic Imaging, Division of Nuclear Medicine and Molecular Imaging, Sidra Medicine, Al-Luqta Street, PO Box Number. 26999, Doha, Qatar.

Abstract: (159 words)

Background: MIBG imaging has been the standard for Neuroblastoma staging following its discovery 4 decades ago. Novel agents such as ${ }^{18}$ F-FDOPA and ${ }^{68}$ Ga-DOTATATE are being widely used nowadays in academic centers. In the midst of the COVID-19 pandemic, procurement of MIBG has proved particularly challenging, necessitating the use of ${ }^{68}$ Ga DOTATATE positron emission tomography.

Methods: ${ }^{68} \mathrm{Ga}$-DOTATATE PET/CT imaging was carried out for completion of staging investigation for three pediatric patients who were diagnosed with Neuroblastoma at our institution, as ${ }^{123}$ I-MIBG became unavailable during the pandemic.

Results: ${ }^{68} \mathrm{Ga}$-DOTATATE PET/CT scans were carried out in all three pediatric patients without any complications. All patients showed ${ }^{68}$ Gallium DOTATATE avid disease. PET/CT scans showed better spa- 
tial resolution, increased lesion contrast and demonstrated high accuracy in staging Neuroblastoma patients in concordance with current EANM guidelines.

Conclusion: ${ }^{68} \mathrm{Ga}$-DOTATATE PET/CT imaging can be reliably used as an alternative to ${ }^{123} \mathrm{I}-\mathrm{MIBG}$ for staging and evaluation of patients with Neuroblastoma. Impact may be best in high risk disease.

\section{Introduction:}

Neuroblastoma accounts for about $10 \%$ of all solid tumors in pediatric age group and is the most common extracranial solid tumor in pediatric age group ${ }^{1}$. It occurs in the neural crest derivatives and has a varied presentation ranging from self-resolving Stage $4 \mathrm{~S}$ disease to the highly malignant variant associated with extremely poor prognosis ${ }^{2,3}$. Staging evaluation of Neuroblastoma requires morphological imaging including Computerized Tomography (CT) and Magnetic Resonance Imaging (MRI) scans as well as nuclear medicine imaging to look for skeletal metastatic deposits ${ }^{4,5} \cdot{ }^{123}$ I-Metaiodobenzylguanadine (MIBG) is a radiopharmaceutical structurally similar to norepinephrine that concentrates within secretory granules of catecholamine-producing neural crest cells. It can be used for localization of neural crest tumors such as Neuroblastoma, pheochromocytoma, and indeed other Neuro Endocrine Tumors anywhere in the body. It has been the standard for Neuroblastoma staging for 4 decades since its utility was first reported by Geatti et al in $1985^{6,7}$.

Reported sensitivity and specificity of ${ }^{123}$ I-MIBG is $~ 90 \%^{5,8}$. However, as $10 \%$ of Neuroblastomas are MIBG non avid, alternate imaging techniques have been investigated including Positron emission tomography (PET) with 2-deoxy-2-[fluorine-18]fluoro-D-glucose $\left({ }^{18} \mathrm{~F}-\mathrm{FDG}\right)$ and radiolabeled somatostatin analogues such as Octreotide, L-3,4-Dihydroxy-6-[18F] fluorophenylalanine $\left({ }^{18} \mathrm{~F}\right.$-DOPA) and 1,4,7,10-tetraazacyclotetradecane$N, N^{\prime}, N ", N "$ ' -tetraacetic acid (DOTA)-conjugated peptides including ${ }^{68}$ Ga- DOTATATE PET/CT. When combined with PET/CT or MRI, these modalities have been shown to be superior in sensitivity and specificity to MIBG thus having the potential of replacing $\mathrm{MIBG}^{9-12}$.

Somatostatin (SST) is a hypothalamic peptide that inhibits the secretion of pituitary growth hormone, pancreatic and gastric hormones. Somatostatin receptors (SSTR) are variably expressed in Neuroblastoma tumor cells ${ }^{13,14}$. Somatostatin analogues such as DOTATATE have been in clinical use for nearly a decade. Their role is most established in imaging and treatment of adult neuroendocrine tumors (NET) and pheochromocytoma, but also studied in Neuroblastoma in limited settings ${ }^{10,11}$. Role of ${ }^{68}$ Ga- DOTATATE PET/CT has been studied at the time of recurrence and at the therapeutic planning phase within a theranostics paradigm. However, there are very few reports of its role at diagnosis for staging 8,15 .

As MIBG production is limited globally, the SARS-CoV-2 (COVID-19) pandemic has resulted in unacceptable delays in carrying out MIBG scans due to interruption in international air traffic. Its availability became particularly challenging at our institution, we therefore decided to perform ${ }^{68}$ Ga-DOTATATE PET/CT scans for three newly diagnosed patients with Neuroblastoma. This PET agent is locally produced in a nearby center obviating all the delivery logistics issues. Here we report our results with a review of the literature.

\section{Methods:}

Three patients were diagnosed with Neuroblastoma since the beginning of the COVID 19 pandemic in March 2020 .

MIBG scintigraphy had to be cancelled for patient 1 due to radiopharmaceutical not arriving on the scheduled date as a result of the cancellation of flights. In discussion with the clinical team, we decided to carry out ${ }^{68} \mathrm{Ga}$-DOTATATE PET/CT imaging as an alternative. For both the subsequent patients, ${ }^{68} \mathrm{Ga}$ - DOTATATE $\mathrm{PET} / \mathrm{CT}$ was done electively to avoid delay and hazard during the pandemic.

A ${ }^{68}$ Germanium $/{ }^{68}$ Gallium generator was used to produce ${ }^{68} \mathrm{Ga}$ locally, which was radiolabeled with 
DOTATATE. Required quality control tests were performed prior to injecting the patient in accordance with good manufacturing practices (GMP). Following radiopharmaceutical injection an uptake phase of 4560 minutes took place prior to scanning the patient. All 3 patients were scanned on a GE Discovery 690 PET-CT scanner with an axial field of view of $15.7 \mathrm{~cm}$. A low dose CT scan $(80 \mathrm{kVP}, \mathrm{mA}$ dose modulation, ASIR, $0.5 \mathrm{~s}$ rotation time, 40-mm collimation), was performed for attenuation correction and anatomical localization followed by a whole body PET at 4 min per bed acquisition with an 11 slice overlap. All data corrections for scatter, randoms and attenuation were employed as implemented on the scanner front end. Images were reconstructed with time of flight iterative reconstruction algorithm with a correction for point spread function (VPFX - 2 iterations, 28 subsets), and a $3.4 \mathrm{~mm}$ post reconstruction Gaussian filter. The final pixel sizes were $1.56 \times 1.56 \mathrm{~mm}$ with a slice thickness of $3.27 \mathrm{~mm}$. All 3 patients were scanned under general anesthesia (GA). The need for sedation was independent of the type of study, as they would have required GA for MIBG as well. There were no complications.

Results:

Patient 1 is a 23-month old toddler who presented with lower back pain, gradually worsening ataxia and leg weakness over the previous 5 days. Physical examination was initially non-suggestive, but quickly progressed to lower limb weakness and frank paraplegia. His blood investigation were normal and chest X-Ray was suggestive of a soft tissue paravertebral mass at the level of upper thoracic vertebrae. This was followed by MRI scan that demonstrated a paravertebral mass at the level of T2-T4 vertebrae, causing spinal cord compression and edema. He underwent emergency laminotomy and laminoplasty by the neurosurgeons and a de-bulking procedure, which relieved pressure on the spinal cord resulting in return of lower limb function over the next 48 hours. His urinary Vanillylmandellic Acid (VMA)/creatinine ratio and Homovanillic Acid (HVA)/creatinine ratio were mildly elevated. Histopathology of the resected tissue showed poorly differentiated Neuroblastoma. Tumor cytogenetics did not reveal any high-risk features.

On ${ }^{68} \mathrm{Ga}$-DOTATATE PET/CT scan, increased DOTATATE uptake was seen in the left paravertebral mass extending into the posterior mediastinum and posterior chest wall and crossing the midline. Another focus of increased DOTATATE uptake was noted along the right paravertebral aspect of thoracic vertebrae at the level of T2 and T3. Additionally, a calcified distinct posterior mediastinal node with increased DOTATATE uptake was also noted. These findings are summarized in Table 1 and ${ }^{68}$ Ga-DOTATATE imaging is shown in (Fig 1).

Patient 2 is a six-year old girl who presented with a mild intermittent limp over the previous 2 months. She did not have any other symptoms. On physical examination, she was unable to stand straight due to left leg pain and had a mildly tender left side of lower abdomen. X-Ray of her pelvis demonstrated 2 radiolucent lesions in her left femur. Further imaging studies including MRI scan of her abdomen and pelvis demonstrated a large left supra-renal mass and numerous bony metastatic lesions in addition to several enlarged retroperitoneal lymph nodes. Her urinary VMA/Creatinine ratio and HVA/Creatinine ratio were moderately elevated. A core biopsy from the suprarenal mass was carried out under ultrasound guidance. Histopathology of the mass confirmed the diagnosis of poorly differentiated Neuroblastoma, which did not have any high-risk cytogenetic features. Her bone marrow was infiltrated by Neuroblastoma.

On ${ }^{68} \mathrm{Ga}$-DOTATATE PET/CT scan, intense DOTATATE uptake was noted in the large left suprarenal mass with areas of photopenia corresponding to necrosis and calcifications. Uptake was also noted in the right adrenal bed as well as in the right retrocrural region. A few bony skull lesions with increased uptake were noted including the skull base most consistent with disease involvement. Extensive skeletal metastasis along the axial and appendicular skeleton were noted with evidence of bone marrow infiltration. These findings are summarized in Table 1 and ${ }^{68}$ Ga-DOTATATE imaging is shown in (Fig 2).

Patient 3 is a 3.5-year old girl who presented with weight loss, left eye swelling, body aches and constitutional symptoms over several months. She was cachectic, severely malnourished and had left sided proptosis. MRI scan showed a right upper abdominal mass arising from the adrenal gland. She also had numerous liver metastases, enlarged lymph nodes along the lower pole of the left kidney and multiple vertebral lesions. It also 
showed enlarged mediastinal lymph nodes at the level of tracheal bifurcation and left posterior mediastinum. Her bone marrow was infiltrated by malignant cells. Urinary VMA/Creatinine ratio and HVA/Creatinine ratio were grossly elevated. In the presence of elevated catecholamines and highly suggestive bone marrow results, we elected not to carry out tumor biopsy.

${ }^{68} \mathrm{Ga}$-DOTATATE PET/CT scan demonstrated intense heterogeneous uptake in the right adrenal mass with skeletal metastatic deposits and diffuse bone marrow infiltration. It also demonstrated uptake in the thoracic and abdominal lymph nodes seen on MRI scan. Additionally, there were two bony lesions in the skull vault. These findings are summarized in Table 1 and selected representative images on ${ }^{68}$ Ga-DOTATATE imaging are shown in (Fig 3).

\section{Discussion:}

COVID-19 pandemic has posed many unanticipated and unprecedented challenges in healthcare worldwide, including cancer patients ${ }^{16}$. Prior to the pandemic, establishing cancer diagnosis and carrying out the standard staging investigation was not seen to be a potential problem for oncology and nuclear medicine departments, especially in high-income countries. Faced with the logistic difficulty of ${ }^{123}$ I-MIBG procurement, we decided to perform ${ }^{68} \mathrm{Ga}$-DOTATATE PET/CT scans, in the presence of limited but compelling evidence of its utility for initial staging of Neuroblastoma. Our results will add to the evidence base for future studies.

${ }^{68} \mathrm{Ga}$-DOTATATE scans in our patients showed a very high spatial resolution and high accuracy quite concordant with reports in literature ${ }^{8,15,17}$. Although direct comparison with MIBG was not possible, functional information and detection rate was higher than concurrent anatomical imaging.

In Neuroblastoma management, the role of MIBG scintigraphy is not only in staging and response evaluation, presence of MIBG avid disease at diagnosis also predicts response to therapy and persistence of disease after induction chemotherapy is indicative of a poor prognosis ${ }^{18,19}$. However, it is also associated with several logistic disadvantages. In addition to the lengthy process of acquiring the radiopharmaceutical, imaging is carried out at least 24 hours after its administration. Children may require inpatient admission and must repeatedly visit the nuclear medicine department. Also, MIBG can result in the accumulation of unbound Iodine in the thyroid gland. Administration of Lugol's solution is therefore required for adequate thyroid blockade. Finally, MIBG uptake is affected by other seemingly innocuous medications that can affect MIBG uptake and result in poor sensitivity and accuracy of the study ${ }^{20,21}$. Imaging with single-photon emission computerized tomography SPECT-CT requires some logistical and software enhancements in order to achieve a similar whole body acquisition as the PET-CT study. On the other hand ${ }^{68}$ Ga-DOTATATE can be produced locally by using a generator and the patient is injected and imaged on the same day. Additionally, no special preparation is required other than avoiding somatostatin analogues.

Role of ${ }^{68} \mathrm{Ga}$-DOTATATE PET/CT has previously been studied at the time of recurrence and at the therapeutic planning phase within a theranostics paradigm. However, there are very few reports of its role at diagnosis for staging 8,15 . Maurice et al found that in their series of 15 adults with pheochromocytoma and paraganglioma, ${ }^{68} \mathrm{Ga}$-DOTATATE was positive in 5 patients where MIBG was negative, whereas converse was true for only 2 patients ${ }^{22}$. Similarly Naji et al found that in adults with Neural crest tumors, ${ }^{68}$ Ga-DOTATATE showed 10 out of 12 lesions, vs. 5 shown by $\mathrm{MIBG}^{23}$. There is a paucity of data in combined MIBG scintigraphy and PET/CT DOTATATE emission tomography. Krois et al compared the sensitivity and specificity of ${ }^{68} \mathrm{Ga}$-DOTATATE to MIBG and morphological imaging in their series of 11 patients (mixed pediatric and adult cohort). Although, only 5 of 11 patients had a diagnosis of Neuroblastoma, DOTATATE sensitivity was $97 \%$ vs $90 \%$ for $\mathrm{MIBG}^{8}$. In a case report, Agarwal et al reported the utility of ${ }^{68} \mathrm{Ga}$-DOTATATE PET/CT in diagnosis and response evaluation in a 12 year old child with Neuroblastoma ${ }^{15}$.

Two of the three patients undergoing gallium imaging had metastatic disease evaluable on morphological imaging and bone marrow evaluation. All lesions evaluable on MR scans were also avid on ${ }^{68}$ Ga-DOTATATE 
PET/CT imaging affirming the hypothesis that it is highly sensitive for Neuroblastoma.

The effective radiation doses for ${ }^{123} \mathrm{I}-\mathrm{MIBG}$ and ${ }^{68} \mathrm{Ga}$-DOTATATE are $0.013 \mathrm{mSv} / \mathrm{MBq}$ and $0.021 \mathrm{mSv} / \mathrm{MBq}$ respectively ${ }^{24,25}$. Depending on the activity schedule for a $20 \mathrm{~kg}$ pediatric patient using the standardized EANM weight-based activity calculator ${ }^{26}$, (62 MBq for ${ }^{68}$ Ga-DOTATATE and $136 \mathrm{MBq}$ for $\left.{ }^{123} \mathrm{I}-\mathrm{MIBG}\right)$ an approximate $25 \%$ radiation dose saving can be made with the use of ${ }^{68} \mathrm{Ga}$-DOTATATE, based on the assumption that a similar low dose CT is performed in both studies ${ }^{17,}$ 24-27.

${ }^{68}$ Ga-DOTATATE PET-CT has clear advantages compared to ${ }^{123}$ I-MIBG scintigraphy, with the latter suffering from low image quality, difficulties in image quantification, and poor spatial resolution of SPECT (up to $1 \mathrm{~cm}$ using medium energy collimators) as well as thyroid blockade and discontinuation of certain medications. PET presents inherent advantages with better spatial resolution (4-5 $\mathrm{mm}$ ) even with a relatively large positron energy of $1.9 \mathrm{MeV}$, quantified images in $\mathrm{kBq} / \mathrm{ml}$. Practical advantages also include more rapid imaging requiring less sedation as well as no specific patient preparation requirements.

\section{Conclusions:}

${ }^{68} \mathrm{Ga}$-DOTATATE PET/CT imaging can be reliably used as an alternative to MIBG for staging and response evaluation. Impact may be best in high risk disease. It has numerous advantages including higher sensitivity, higher spatial resolution from PET, better image contrast, better tumor-to-background ratio, easier patent flow, easier patient preparation and a more favorable dosimetry profile.

Conflicts of Interest: None of the authors have any conflicts of interest.

Acknowledgements: We would like to thank Cyclomedical-HMC in facilitating the provision of ${ }^{68} \mathrm{Ga}-$ DOTATATE.

\section{Legend List:}

1. (Fig 1): Increased uptake in a left sided paravertebral mass and invading the spinal canal and adjacent vertebral body of T3 (blue arrow). Additional lesions are clearly identified in the right paravertebral space of $\mathrm{T} 3$ and in the posterior mediastinum (white arrow).

2. (Fig 2): Uptake in the left suprarenal mass with areas of necrosis (blue arrow) and evidence of skeletal metastasis (white arrows) and bone marrow infiltration.

3. (Fig 3): Heterogeneous uptake in a lobulated complex abdominal/right suprarenal mass (blue arrow) with diffuse skeletal metastasis including in the skull (white arrows) and evidence of diffuse bone marrow infiltration.

\section{References:}

1. Jiang M, Stanke J, Lahti JM. The connections between neural crest development and Neuroblastoma. Curr Top Dev Biol . 2011;94:77-127.

2. Brodeur GM, Pritchard J, Berthold F, et al. Revisions of the international criteria for Neuroblastoma diagnosis, staging, and response to treatment. Journal of Clinical Oncology . 1993;11(8):1466-1477.

3. Maris JM, Hogarty MD, Bagatell R, Cohn SL. Neuroblastoma. The Lancet . 2007/06/23/ 2007;369(9579):2106-2120.

4. Monclair T, Brodeur GM, Ambros PF, et al. The International Neuroblastoma Risk Group (INRG) staging system: an INRG Task Force report. J Clin Oncol . Jan 10 2009;27(2):298-303.

5. Bar-Sever Z, Biassoni L, Shulkin B, et al. Guidelines on nuclear medicine imaging in Neuroblastoma. European Journal of Nuclear Medicine and Molecular Imaging . 2018/10/01 2018;45(11):2009-2024.

6. Geatti O, Shapiro B, Sisson JC, et al. Iodine-131 metaiodobenzylguanidine scintigraphy for the location of Neuroblastoma: preliminary experience in ten cases. J Nucl Med . Jul 1985;26(7):736-42. 
7. Smets LA, Janssen M, Metwally E, Loesberg C. Extragranular storage of the neuron blocking agent metaiodobenzylguanidine (MIBG) in human Neuroblastoma cells. Biochem Pharmacol . Jun 15 1990;39(12):195964 .

8. Kroiss A, Putzer D, Uprimny C, et al. Functional imaging in phaeochromocytoma and Neuroblastoma with 68Ga-DOTA-Tyr3-octreotide positron emission tomography and 123I-metaiodobenzylguanidine: a clarification. European Journal of Nuclear Medicine and Molecular Imaging . 2012/03/01 2012;39(3):543-543.

9. Bleeker G, Tytgat GA, Adam JA, et al. 123I-MIBG scintigraphy and 18F-FDG-PET imaging for diagnosing Neuroblastoma. Cochrane Database Syst Rev . Sep 29 2015;2015(9):Cd009263.

10. Kong G, Hofman MS, Murray WK, et al. Initial Experience With Gallium-68 DOTA-Octreotate PET/CT and Peptide Receptor Radionuclide Therapy for Pediatric Patients With Refractory Metastatic Neuroblastoma.Journal of Pediatric Hematology/Oncology . 2016;38(2):87-96.

11. Gains JE, Bomanji JB, Fersht NL, et al. 177Lu-DOTATATE molecular radiotherapy for childhood Neuroblastoma. J Nucl Med. Jul 2011;52(7):1041-7.

12. Shulkin BL, Hutchinson RJ, Castle VP, Yanik GA, Shapiro B, Sisson JC. Neuroblastoma: positron emission tomography with 2-[fluorine-18]-fluoro-2-deoxy-D-glucose compared with metaiodobenzylguanidine scintigraphy. Radiology. Jun 1996;199(3):743-50.

13. O'Dorisio MS, Chen F, O'Dorisio TM, Wray D, Qualman SJ. Characterization of somatostatin receptors on human Neuroblastoma tumors. Cell Growth Differ. Jan 1994;5(1):1-8.

14. Albers AR, O'Dorisio MS, Balster DA, et al. Somatostatin receptor gene expression in Neuroblastoma. Regul Pept. Mar 17 2000;88(1-3):61-73.

15. Agrawal K, Kumar R, Shukla J, Bhattacharya A, Mittal BR. Ga-68 DOTATATE positron emission tomography/computer tomography in initial staging and therapy response evaluation in a rare case of primary Neuroblastoma in neck. Indian J Nucl Med . 2014;29(3):175-176.

16. Sullivan M, Bouffet E, Rodriguez-Galindo C, et al. TITLE: The COVID-19 PANDEMIC: A Rapid Global response for Children with Cancer from SIOP, COG, SIOP-E, SIOP-PODC, IPSO, PROS, CCI and St Jude Global.Pediatric Blood \& Cancer . 04/01 2020.

17. Alexander N, Vali R, Ahmadzadehfar H, Shammas A, Baruchel S. Review: The Role of Radiolabeled DOTA-Conjugated Peptides for Imaging and Treatment of Childhood Neuroblastoma. Curr Radiopharm . 2018 2018;11(1):14-21.

18. Suc A, Lumbroso J, Rubie H, et al. Metastatic Neuroblastoma in children older than one year: Prognostic significance of the initial metaiodobenzylguanidine scan and proposal for a scoring system.Cancer . 1996;77(4):805-811.

19. Lewington V, Lambert B, Poetschger U, et al. (123)I-mIBG scintigraphy in Neuroblastoma: development of a SIOPEN semi-quantitative reporting, method by an international panel. Eur J Nucl Med Mol Imaging . Feb 2017;44(2):234-241.

20. Van Vickle SS, Thompson RC. 123I-MIBG Imaging: Patient Preparation and Technologist's Role. $J$ Nucl Med Technol. Jun 2015;43(2):82-6.

21. Sharp SE, Trout AT, Weiss BD, Gelfand MJ. MIBG in Neuroblastoma Diagnostic Imaging and Therapy. Radiographics . Jan-Feb 2016;36(1):258-78.

22. Maurice JB, Troke R, Win Z, et al. A comparison of the performance of 68Ga-DOTATATE PET/CT and 123I-MIBG SPECT in the diagnosis and follow-up of phaeochromocytoma and paraganglioma. European Journal of Nuclear Medicine and Molecular Imaging . 2012/08/01 2012;39(8):1266-1270. 
23. Naji M, Zhao C, Welsh SJ, et al. 68Ga-DOTA-TATE PET vs. 123I-MIBG in identifying malignant neural crest tumours. Mol Imaging Biol . Aug 2011;13(4):769-75.

24. Bombardieri E, Giammarile F, Aktolun C, et al. 131I/123I-metaiodobenzylguanidine (mIBG) scintigraphy: procedure guidelines for tumour imaging. Eur J Nucl Med Mol Imaging . Dec 2010;37(12):2436-46.

25. Sandström M, Velikyan I, Garske-Román U, et al. Comparative biodistribution and radiation dosimetry of 68Ga-DOTATOC and 68Ga-DOTATATE in patients with neuroendocrine tumors. $J$ Nucl Med . Oct 2013;54(10):1755-9.

26. Jacobs F, Thierens H, Piepsz A, et al. Optimised tracer-dependent dosage cards to obtain weightindependent effective doses.European Journal of Nuclear Medicine and Molecular Imaging . 2005/04/01 2005;32(5):581-588.

27. Walker RC, Smith GT, Liu E, Moore B, Clanton J, Stabin M. Measured human dosimetry of 68GaDOTATATE. J Nucl Med . Jun 2013;54(6):855-60.

\section{Hosted file}

Gallium Dotatate Manuscript Table.docx available at https://authorea.com/users/337025/ articles/462692-use-of-68ga-dotatate-pet-ct-imaging-for-staging-evaluation-ofneuroblastoma-in-the-covid-19-pandemic-potential-for-clinical-use 
Figure 1. Selected representative images of ${ }^{68}$ Ga-DOTATATE PET/CT for Patient 1

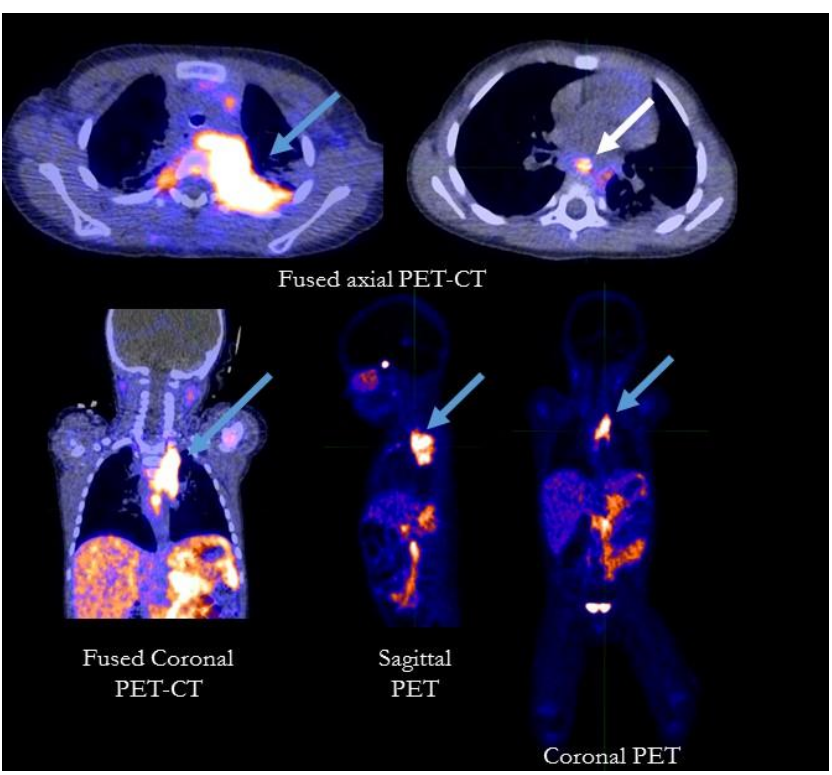

Increased uptake in a left sided paravertebral mass and invading the spinal canal and adjacent vertebral body of T3 (blue arrow). Additional lesions are clearly identified in the right paravertebral space of T3 and in the posterior mediastinum (white arrow). 
Figure 2. Selected representative images of ${ }^{68} \mathrm{Ga}$-DOTATATE PET/CT for Patient 2

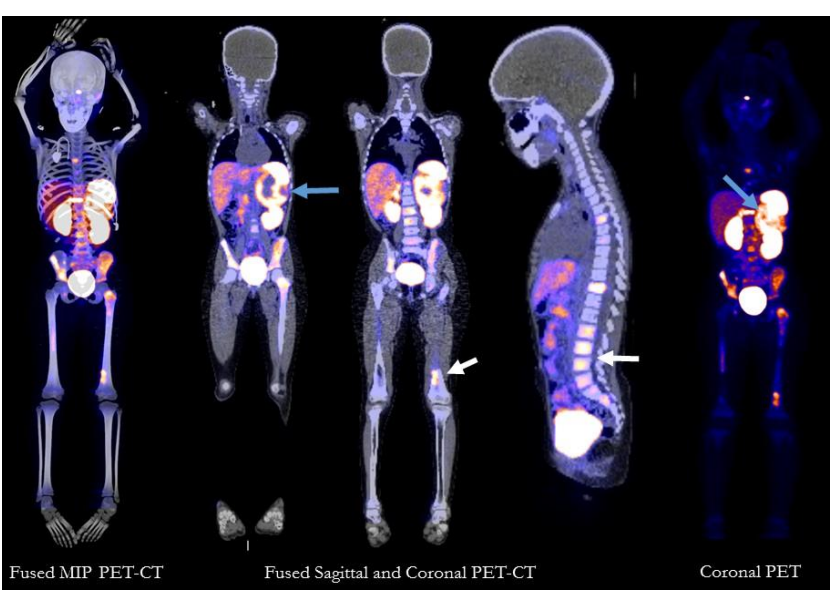

Uptake in the left suprarenal mass with areas of necrosis (blue arrow) and evidence of skeletal metastasis (white arrows) and bone marrow infiltration. 
Figure 3. Selected representative images of ${ }^{68}$ Ga-DOTATATE PET/CT for Patient 3

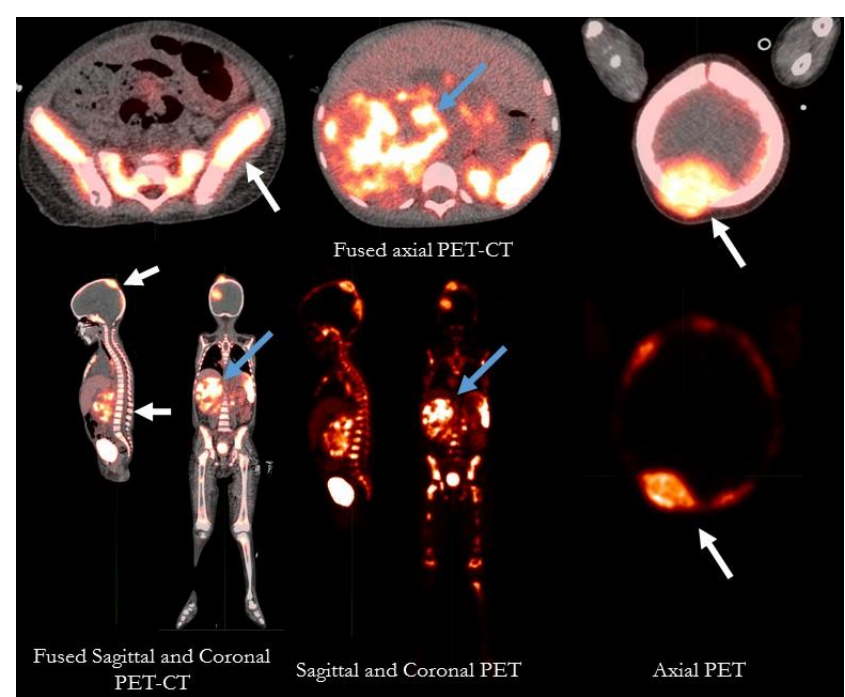

Heterogeneous uptake in a lobulated complex abdominal/right suprarenal mass (blue arrow) with

diffuse skeletal metastasis including in the skull (white arrows) and evidence of diffuse bone marrow infiltration. 